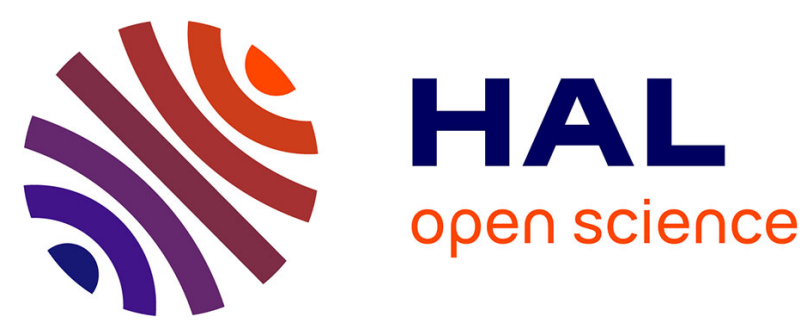

\title{
De la lutte armée à la nation palestinienne. Vers une relecture des rapports entre l'OLP et les réfugiés
}

\author{
Jalal Al Husseini, Kamel Doraï
}

\section{To cite this version:}

Jalal Al Husseini, Kamel Doraï. De la lutte armée à la nation palestinienne. Vers une relecture des rapports entre l'OLP et les réfugiés. Autrepart - Revue de sciences sociales au Sud, 2003, 26, pp.91-106. 10.3917/autr.026.0091 . halshs-00293573

\section{HAL Id: halshs-00293573 \\ https://shs.hal.science/halshs-00293573}

Submitted on 4 Jan 2009

HAL is a multi-disciplinary open access archive for the deposit and dissemination of scientific research documents, whether they are published or not. The documents may come from teaching and research institutions in France or abroad, or from public or private research centers.
L'archive ouverte pluridisciplinaire HAL, est destinée au dépôt et à la diffusion de documents scientifiques de niveau recherche, publiés ou non, émanant des établissements d'enseignement et de recherche français ou étrangers, des laboratoires publics ou privés. 


\title{
De la lutte armée à la nation palestinienne. \\ Vers une relecture des rapports entre l'OLP et les réfugiés.
}

\author{
Jalal Al Husseini - CERMOC, Amman \\ Mohamed Kamel Doraï - CERMOC, Amman
}

\section{Introduction.}

Depuis cinquante ans les conflits se succèdent au Moyen-Orient et l'actualité confirme la permanence des tensions dans la région. La question palestinienne se trouve au cœur de ces tensions. La liste est longue des conflits qui ont marqué la société palestinienne : l'exode de 1948, la Guerre des Six jours en 1967, les guerres du Liban qui ont déchiré ce pays entre 1975 et 1991 et aujourd'hui la deuxième intifada déclenchée en septembre 2001. Ces guerres n'ont pas seulement affecté la société palestinienne mais ont bouleversé sa structure sociale, donnant naissance à l'une des populations de réfugiés les plus anciennes et les plus nombreuses de la deuxième moitié du vingtième siècle. On compte effectivement aujourd'hui plus de 3500000 réfugiés palestiniens au Moyen-Orient. La dispersion liée au conflit israéloarabe de 1948, la lutte armée et son influence déterminante dans la structuration du mouvement national palestinien, ont entrainé une redéfinition profonde de la société palestinienne. Quelles nouvelles formes d'identification et de solidarités ont émergé de cette nouvelle configuration socio-spatiale imposée par la guerre ?

Une attention particulière est portée dans un premier temps sur le rôle joué par les camps de réfugiés dans le maintien de l'identité palestinienne ainsi que la place centrale occupée par la Palestine, comme symbole, dans la structuration de la diaspora. Dans un deuxième temps, c'est l'organisation de la diaspora qui est analysée en rapport avec l'importance croissante occupée par la lutte armée dans le discours produit par la Résistance palestinienne. Nous nous appuierons principalement sur le cas des Palestiniens du Liban, qui du fait du rôle que l'Organisation de Libération de la Palestine (OLP) y a joué, éclaire de façon significative l'importance des conflits et de la lutte armée dans la construction de la société palestinienne d'aujourd'hui. La présence palestinienne au Liban est effectivement marquée par la guerre. L'arrivée des réfugiés fait suite à l'exode des Palestiniens durant la guerre israélo-arabe de 1948. Quelques années plus tard, la guerre civile libanaise en 1975 puis l'invasion israélienne de 1982 détruisent l'essentiel des infrastructures palestiniennes. La guerre des camps (1985- 
1987) achève cette entreprise et les réfugiés palestiniens se trouvent marginalisés sur la scène socio-politique libanaise. Le présent travail se base sur le partage d'une expérience de terrain acquise depuis le milieu des années quatre-vingt-dix, et d'une relecture des textes de la résistance palestinienne.

\section{Les camps de réfugiés palestiniens: conflits, exodes et mémoire.}

Si la création de l'Etat d'Israël en 1948 a répondu aux aspirations du mouvement sioniste à la suite de la Seconde Guerre Mondiale, elle a entraîné la déstructuration de la société palestinienne et jeté sur les routes de l'exil près de 750000 réfugiés [Mission économique d'études des Nations Unies pour le Moyen-Orient, 1949]. Il existe aujourd'hui une soixantaine de camps de réfugiés administrés par l'UNRWA ${ }^{1}$ disséminés dans les territoires palestiniens ainsi que dans les pays arabes alentours. Cette agence internationale joue un rôle de support matériel fondamental (distribution de rations, construction d'habitat, services sociaux, etc.) dans le contexte de la survie et de la réhabilitation de la population palestinienne, même si cette assistance induit une inévitable dépendance envers ces différents services. Quelle relecture de l'identité palestinienne la dispersion et la présence des camps de réfugiés impliquent-elles ? L'espace des camps remplit quatre fonctions principales : c'est un lieu de mémoire et d'affirmation identitaire, un lieu d'habitat, un espace de développement ou de repli économique pour les réfugiés, ainsi qu'un lieu d'exercice et de développement du pouvoir politique et armé.

\subsection{Conflit, exode et genèse des camps de réfugiés.}

Suite au conflit de 1948, les Palestiniens se retrouvent sans terre, privés d'Etat ${ }^{2}$, dispersés à travers le Moyen-Orient et plus loin encore pour certains. La nakba, catastrophe en arabe, qui

\footnotetext{
1 UNRWA : Office de secours et de travaux des Nations Unies pour les réfugiés de Palestine dans le ProcheOrient, créé en décembre 1949 par l'ONU pour subvenir aux besoins des réfugiés de Palestine dans les domaines de l'éducation, de la santé, des secours et des services sociaux. Il officie aujourd'hui en Cisjordanie, Gaza, Jordanie, Liban et Syrie.

2 La résolution 181, votée le 29 novembre 1947 par l'Assemblée Générale de l'ONU, propose la partition de la Palestine en trois entités qui doivent devenir indépendantes le $1^{\text {er }}$ août 1948 : deux Etats, l'un Juif, l'autre arabe, ainsi qu'une zone sous contrôle international, qui englobe Jérusalem et les localités voisines, gérée par l'ONU. A l'issu de guerre israélo-arabe de 1948, Israël voit le jour et fait son entrée à l'ONU, alors que la bande de Gaza passe sous administration égyptienne et que la Cisjordanie est annexée par la Transjordanie voisine. Suite à la Guerre des Six jours en juin 1967, l'ensemble du territoire de la Palestine mandataire se retrouve sous autorité israélienne.
} 
désigne pour les Palestiniens l'exode de 1948, marque durablement le peuple palestinien. Depuis leur exil, les réfugiés développent leur identité propre, et affirment leur droit au retour. Leur détermination est à la hauteur de l'évènement subi. Le territoire perdu revit alors au sein de chaque communauté dispersée, et la terre palestinienne devient le symbole d'un peuple entier, comme si l'éloignement renforçait encore plus l'attachement à la terre palestinienne toute entière. L'Etat d'Israël se développe de son côté et son empreinte sur l'espace palestinien est de plus en plus marquée. Ses frontières s'agrandissent en 1967, et l'Etat s'affirme comme une réalité pérenne, avec laquelle les Palestiniens et les Etats arabes doivent désormais composer. De la dispersion des Palestiniens naît la diaspora ${ }^{3}$, qui plonge ses racines dans la construction nationale édifiée depuis l'époque ottomane et qui se cristallise dorénavant autour des centralités symboliques du territoire perdu et de Jérusalem. Depuis plus de cinquante ans aujourd'hui, la question des réfugiés demeure en suspend. L'enjeu démographique et politique qu'elle suscite pour Israël représente depuis 1948 un obstacle à la paix ainsi qu'à une solution juste pour les réfugiés. D'une réalité provisoire à leur création, les camps de réfugiés s'inscrivent donc durablement dans leurs espaces d'accueil alors que le conflit israélo-arabe s'enlise.

Dans un premier temps, les Palestiniens dispersés se sont dirigés vers les camps de réfugiés pour différents motifs. La proximité spatiale du lieu de leur d'arrivée ainsi que les facteurs économiques sont déterminants pour comprendre la répartition géographique actuelle des réfugiés palestiniens. De nombreux réfugiés sont arrivés au Liban sans aucune ressource, ils se sont donc dirigés vers les camps de réfugiés les plus proches de leur lieu d'arrivée pour se loger et pour se nourrir. D'autres réfugiés se sont installés dans les camps plus tardivement, lorsqu'à cours d'argent ils ne pouvaient plus louer un logement dans les villes ou villages libanais. En témoigne une femme âgée du camp d'Al Buss, originaire d'Oum al Faraj en Palestine $^{4}$ :

"A notre arrivée, nous sommes venus habiter à Tyr, dans la ville. Notre père était riche, il ne voulait pas nous mettre dans les camps, dans la saleté. Ici il y avait des Arméniens avant. Ils

\footnotetext{
3 Gabriel Sheffer [1996] propose trois critères pour définir une diaspora. Tout d'abord le maintien et le développement d'une identité propre au peuple en diaspora. En deuxième lieu, les membres de la diaspora doivent entretenir des contacts significatifs avec leur patrie d'origine, sous forme réelle ou symbolique. En dernier lieu, la diaspora doit disposer d'une organisation interne distincte de celle de son Etat d'origine ou d'accueil.

${ }^{4}$ Les entretiens qui suivent ont été réalisés par M. K. Doraï entre 1999 et 2001 au Liban. Les traductions sont de l'auteur.
} 
sont partis vers Beyrouth. Après avoir dépensé tout l'argent que nous avions, notre famille est venue ensuite habiter à Al Buss."

D'autres réfugiés ont privilégié les regroupement familiaux et villageois, sans réelle motivation économique. L'efficacité du regroupement villageois est locale, c'est-à-dire que dans chaque région d'arrivée, les réfugiés se sont regroupés par village d'origine. Il existe cependant des personnes originaires d'un même village qui résident dans plusieurs camps de réfugiés au Liban, la guerre civile libanaise qui débute en 1975 a effectivement entraîné d'importants déplacements de population. Par exemple, les Palestiniens originaires du village de Damoun en Palestine et qui sont arrivés à Tyr se trouvent en grande partie dans le camp d'Al Buss comme en témoigne un de ses habitants :

"Je suis né à Damoun, dans la région d'Acre, en 1932. Nous, les gens de Damoun, nous sommes partis de Palestine en 1948, et nous nous sommes installés en premier dans un village qui s'appelle Bazoulieh, au sud Liban. Nous sommes restés là 6 ans. Ensuite, nous sommes venus vers Al Buss parce qu'ici il y avait un camp de réfugiés. La seule raison est que nous avions des proches qui habitaient là."

La vie dans les camps représente dans une certaine mesure une amélioration des conditions de vie des réfugiés. Après de longs mois, voire des années, passés sans logement ou dans des conditions d'habitat très précaires - les récits de réfugiés qui ont vécu dans des abris naturels ou des logements de fortunes dans la nature sont nombreux, tant au Liban qu'en Jordanie l'installation dans les camps permet d'obtenir une tente ainsi que des services de bases (nourriture, médecin, aide sociale). En 1959, l'UNRWA indique que l'ensemble des tentes ont disparu des camps et que l'habitat en dur s'y est substitué [UNRWA, 1986]. Il n'en demeure pas moins que la vie dans ces camps est très dure, du fait de leur insalubrité et de l'absence de revenus stables pour leurs habitants.

\subsection{Une identité palestinienne ancrée dans les camps de l'exil.}

L'identité palestinienne qui s'est construite dans l'exil est fortement ancrée dans les camps de réfugiés qui en sont l'une des incarnations spatiales les plus visibles et les plus représentatives. Les camps palestiniens représentent trois aspects de l'identité palestinienne en exil : (1) la permanence territoriale, -c'est un lieu de stabilité et de continuité malgré son caractère temporaire par définition -. (2) Un lieu de "l'entre-soi", la concentration spatiale des Palestiniens facilite la reproduction partielle des systèmes de solidarité villageois et familiaux. 
Les camps de réfugiés jouent donc un rôle actif dans l'organisation et le développement des réseaux sociaux palestiniens. Ils peuvent alors être considérés en partie comme des "espaces mémoires" de la diaspora, où s’inscrivent l'histoire et la généalogie palestinienne. (3) Un espace d'échanges avec les sociétés d'accueil, mais aussi de conflit comme au Liban pendant la guerre civile ou en Jordanie durant Septembre Noir.

L'organisation spatiale des camps, leur seule présence effective, la permanence avérée de leur implantation, font revivre pour les réfugiés la Palestine dans l'exil. Les images spatiales jouent un grand rôle dans la construction de la mémoire collective. Il existe une dialectique entre espace et groupe social. L'espace est une donnée immuable dans le temps qui sert de repère pour la mémoire. Comme le montre Martine Hovanessian [1995], se référer à un espace, c'est affirmer la persistance de son identité à travers le temps. Faire appel à l'espace, c'est une manière de faire resurgir le passé dans le présent, en donnant l'impression que le passé n'est pas altéré par le temps.

En s'installant dans les camps, les Palestiniens inscrivent dans le paysage leur territoire perdu. Ils délimitent l'intérieur et l'extérieur de leur territoire. Ce dernier sédimente les étapes de la migration, et fait côtoyer le proche et le lointain, les solidarités villageoises et les pratiques sociales nouvelles [Hovanessian, 1995]. C'est par ce processus qu'on assiste pour les Palestiniens de la diaspora à une transposition de la référence spatiale du village vers le camp. Le lieu de vie actuel est investi du territoire d'origine. Il représente en même temps le lieu de pérennité de l'identité (rappel du village d'origine, présence d'images de la Palestine, de drapeaux, etc.) et de l'affirmation de l'identité nouvelle. "Le camp continue ainsi à remplir la fonction jadis assumée par le village, à savoir le maintien d'un équilibre moral des individus qui le composent, mais à un niveau d'organisation et à une échelle démographique d'un ordre supérieur, celle de l'identité nationale palestinienne" [Ghazzawi, 1989 : 37]. La vivacité de la mémoire palestinienne est perceptible dans le discours produit par les réfugiés comme en témoigne des enfants de la troisième génération d'exilés, qui donnent le nom du village de leurs grands-parents lorsqu'on leur demande d'où ils viennent. Réponse d'autant plus intéressante que ces villages ont depuis longtemps été rasés, et n'existent plus que dans les souvenirs des réfugiés. Le rappel et la reconstitution du paysage palestinien dans les camps de réfugiés, par le biais de photographies ou par l'entretien d'un minuscule jardin ont un sens. Le plus souvent il s'agit de quelques plantes ou quelques animaux de basse-cour. 
Dans le camp d'Al Buss, près de la ville de Tyr au sud Liban, lorsque l'espace entre les habitations le permet, les réfugiés d'origine rurale entretiennent le plus souvent des jardins potagers. Quelques fois un olivier qui a été ramené de Palestine, lors des rares visites autorisées durant l'invasion israélienne de 1982, est planté au milieu de la cours intérieure. Ces éléments paysagers, s'ils ont une utilité alimentaire et économique, participent aussi à la reconstruction symbolique du caractère rural de la Palestine d'avant 1948. Augustin Berque [1990 : 79] note à ce propos que "les discours sur le paysage sont souvent des discours métaphoriques sur l'identité nationale, et fleurissent-ils quand celle-ci veut s'affirmer".

Il faut relever l'importance du discours produit par les réfugiés sur l'exil et sur leur vision de la Palestine. Une attention particulière doit être accordée à l'histoire orale de l'exode, qui transmise de génération en génération, tient lieu de véritable récit de la nakba. L'histoire orale transmet aussi la terre et les paysages palestiniens. Les images de Palestine sont gravées dans la mémoire des jeunes générations avec une grande précision. L'histoire orale joue le rôle d'un réservoir identitaire, où les images du passé sont relues et embellies à la lumière des difficultés que vivent actuellement nombre de Palestiniens. Un réfugié âgé d'origine rurale qui vient de Galilée et qui réside dans le camp d'Al Buss raconte la vie en Palestine :

"En Palestine tout poussait sans problème, il n'y avait qu'à planter... La vie était belle, nous avions nos terres que nous cultivions pour manger et nous vendions les surplus sur les marchés. Rien n'était vraiment cher. La solidarité villageoise était vraiment forte, tout le monde s'entraidait. Maintenant tout cela a changé, ici le travail est dur et nous ne vivons pas bien."

L'histoire orale est produite essentiellement au sein de la cellule familiale. Les récits de vie sont un des matériels d'étude privilégiés, car ils permettent d'accéder à la part non écrite de l'histoire ainsi qu'aux trajectoires individuelles et familiales.

\subsection{Les camps de réfugiés lieux d'expression de la mémoire familiale et villageoise.}

La mémoire familiale se transmet oralement par la narration de l'exode et les rappels du déroulement de la vie passée. La vie dans les camps se justifie alors par l'histoire de l'exode et l'espace du camp prend alors un sens pour ses habitants. Le camp n'est plus seulement un lieu de vie, mais devient paradoxalement le symbole de l'exode, en ce sens qu'il en est l'incarnation spatiale, comme si chaque Palestinien avait transporté avec lui un morceau de sa 
terre et qu'il l'avait déposé là, recréant dans son espace de vie un morceau de Palestine. C'est ce qui fonde les rapports entre mémoire et territoire. Les lieux ont une signification pour les individus qui les fréquentent, ce sont des lieux de mémoire. "Alors même qu'on pourrait croire qu'il en est autrement, quand les membres d'un groupe sont dispersés et ne retrouvent rien, dans leur nouvel entourage matériel, qui leur rappelle la maison et les chambres qu'ils ont quittées, s'ils restent unis à travers l'espace, c'est qu'ils pensent à cette maison et à ces chambres" [Halbwachs, 1950]. En retour, les sociétés impriment leur mémoire et leur culture dans l'espace en se l'appropriant. Dans le cas palestinien, ceci est facilité par les regroupements des réfugiés en fonction de leur village et région d'origine. Les différentes parties des camps de réfugiés portent souvent les noms des villages d'origines des réfugiés en Palestine. Nommer l'espace permet aux réfugiés de se l'approprier. Les noms de villages donnés aux différentes parties des camps marquent ainsi la présence effective de la Palestine perdue dans le lieu de vie des réfugiés. Le plus souvent, ces toponymes n'existent plus que dans l'exil, les villages d'origines ayant été détruits ou renommés par Israël. Le quartier ainsi nommé continue de faire vivre dans le quotidien le village d'origine, on peut se rendre par exemple à Al Buss dans le hay damouni [en arabe, quartier des habitants de Damoun], à l'ouest du camp. Les Palestiniens accordent une grande importance à ces lieux de mémoire, qui leur permettent de s'inscrire dans une continuité par rapport au territoire palestinien, et leur donnent le sentiment de la perpétuation de la vie villageoise d'avant 1948. La nomination des quartiers constitue alors une forme d'appropriation de l'espace, qui matérialise son identité.

Le souvenir de ces villages, leur transposition dans l'exil, confèrent aux réfugiés de la diaspora un rôle actif dans la préservation de la géographie palestinienne, ils deviennent les principaux détenteurs de l'histoire de nombreux villages, et les témoins principaux de leur existence. Par exemple, il existe à Qasmiyyeh - un camp informel dans la région de Tyr ${ }^{5}$-, une association qui regroupe les Palestiniens originaires du village d'Al khalsa, au nord de Safed. De ce village il ne reste aujourd'hui que les ruines de quelques bâtiments administratifs, de la mosquée et de l'école de l'époque du mandat. La colonie de Qiryat Shémona s'est développée sur le site de l'ancien village palestinien. L'association des Palestiniens d'Al khalsa tente pourtant de continuer de faire vivre ce village dans l'exil, comme en témoigne un de ses membres :

\footnotetext{
${ }^{5}$ Les camps informels sont des espaces non gérés par l'UNRWA où résident des réfugiés.
} 
"Nous avons fait cette année [1999] un calendrier avec le dessin de notre école à Al khalsa. Nous l'avons dessinée en fonction de notre souvenir, mais c'est elle exactement. Pour que les enfants sachent que nous avions une école là. C'est pour nous souvenir. C'est pour que nos enfants, nos petits enfants aient envie de revenir en Palestine."

La mémoire palestinienne se lit également dans la géographie des camps établis au Liban. On retrouve dans les camps et les groupements palestiniens une organisation socio-spatiale qui rappelle celle des villages d'origine. Les réfugiés tentent le plus souvent de se regrouper par famille et par village d'origine. Ces regroupements sont cependant tributaires des déplacements liés à la guerre et tendent à perdre peu à peu de leur caractère systématique. La reconstruction de la géographie palestinienne peut se lire à grande échelle, celle des camps de réfugiés. Par exemple, l'organisation interne du camp de Borj Shémali est dominée par les regroupements villageois. Les quartiers du camp s'organisent par village d'origine en Palestine (Fig. 1). Des quartiers contigus comme ceux de Chouq et Naameh, correspondent à des villages proches dans le doigt de Galilée. D'autres quartiers mitoyens comme celui de Dichoum et Husseiniyyeh, correspondent à deux villages de Maghrabi ${ }^{6}$, qui se sont regroupés ici par affinité et proximité socio-historique. Ce type de regroupement n'est cependant pas systématique, chaque quartier peut en effet être composé de réfugiés d'origines diverses. Seuls les villages dont un grand nombre de familles résident dans ce camp ont pu se regrouper. La proximité spatiale permet la reconstruction partielle des systèmes de solidarités villageoises qui existaient avant 1948 en Palestine, ainsi que la perpétuation des pouvoirs locaux traditionnels comme ceux du moukhtar, qui peut exercer une autorité morale dans un certain nombre de cas. Dans les autres camps de la région de Tyr on ne peut trouver de regroupements villageois aussi systématiques, la dispersion des familles de même origine est plus grande, et l'on ne retrouve que quelques quartiers qui concentrent une forte proportion de familles venues du même village en Palestine.

A une plus grande échelle, c'est l'habitat qui joue le rôle de lieu de permanence de la société ainsi que de l'identité palestinienne en exil. La fonction identitaire ne doit cependant pas faire oublier les conditions précaires de l'habitat dans les camps et les groupements ainsi que le manque d'infrastructures.

\footnotetext{
${ }^{6}$ Les Maghrabi sont une communauté composée d'Arabes et de Berbères qui ont quitté l'Algérie avec l'Emir Abdel Qader, après avoir résisté contre la colonisation française. Les Ottomans leurs ont donné des terres en Egypte, en Palestine et en Syrie.
} 


\subsection{Les camps de réfugiés au Liban, un espace communautarisé.}

Alain Médam [1992] relève que l'individu en diaspora doit savoir vivre parmi les autres sans vivre tout à fait avec eux. C'est cette dialectique intégration / distanciation qui crée les limites de l'espace diasporique et donne à la diaspora sa dynamique interne. "Etre avec" sans perdre son individualité permet à la diaspora de conserver son indépendance et de se redéployer lorsque son existence est mise en danger. Cette dualité de la culture diasporique donne naissance à une pratique particulière du territoire. "De manière de plus en plus forte, pour chacun dans la diaspora, tout autant qu'il est impossible de vivre entièrement un territoire quelconque - de l'assumer pleinement et sans réserve - il s'avère impossible de ne pas vivre simultanément - bien que partiellement chaque fois - des territoires distincts et éloignés les uns des autres" [Médam, 1992 : 16]. C'est pour cela que le camp de réfugiés est vécu par les Palestiniens comme une réalité transitoire, passagère, bien qu'elle dure depuis plus de cinquante ans aujourd'hui. Les réfugiés ne peuvent se résigner à s'installer durablement et vivre pleinement dans l'espace où ils résident. Cet aspect dépend aussi de la politique menée par le pays d'accueil, en Jordanie par exemple, les Palestiniens ont obtenu pour la plupart la nationalité jordanienne ce qui leur garanti la pérennité de leur présence. Dans le même temps, les habitants du camp vivent là dans une Palestine exilée, l'espace du camp rassemblant des fragments de Palestine, comme les pièces d'un puzzle dispersées qui retrouveraient là, dans cet autre lieu, une unité certaine.

Si à Amman, ou à Damas, on peut observer une certaine intégration des camps de réfugiés dans le tissu urbain et économique de ces villes, qui leur donne une certaine invisibilité, au Liban les camps de réfugiés palestiniens sont beaucoup moins bien intégrés dans le tissu urbain. Ils sont pour certains d'entre eux, comme Chatila à Beyrouth, facilement reconnaissables, et se trouvent isolés spatialement au sud Liban.

L'espace des camps palestiniens apparaît donc comme un lieu d'expression de la singularité de la communauté palestinienne, voulue par les acteurs palestiniens, comme un lieu d'expression de leur histoire particulière, et comme lieu de pérennité de leur identité. C'est aussi un espace maîtrisé, contrôlé aujourd'hui par l'Etat libanais. L'espace des camps palestiniens peut être considéré comme la face visible de la communautarisation palestinienne au Liban. Ils ont aussi été à maintes reprises des espaces de conflits avec les sociétés d'accueil, qui, en 
s'attaquant aux camps de réfugiés, ont tenté de déstabiliser la société palestinienne dans son ensemble, comme cela a été le cas durant Septembre Noir en Jordanie (1970) à Sabra et Chatila (1982) ou pendant la Guerre des camps au Liban (1985-1987). Pour reprendre l'expression de Rosemary Sayigh [1994], les Palestiniens - plus particulièrement au Liban se sont confrontés à de trop nombreux ennemis. Cette communautarisation a atteint son apogée durant la Guerre des camps qui a opposé la milice chiite libanaise Amal aux Palestiniens. Les camps de Chatila à Beyrouth et Rashidiyyeh à Tyr ont été soumis à un blocus total destiné à provoquer le départ de leurs habitants. Un habitant de Rashidiyyeh témoigne :

"La milice Amal a commencé à encercler le camps de tous les côtés. Ils contrôlaient les entrées et les sorties de toutes les personnes dans le camp, c'était en 1985. Ensuite la situation politique s'est durcie dans les camps. Amal ne laissait plus personne entrer dans le camp, pas même les médecins. Le siège autour du camp était complet. Les médias ont alors répandu cette idée de guerre des camps, et ont installé un réel climat de peur et de combat dans les camps et au Liban en général. Ils ont contribué à dresser les communautés les unes contre les autres. Il y a eu plus de cent trente morts dans ce camp, surtout des femmes, des enfants et des vieillards et près de quatre cents blessés. On voyait les gens errer dans les rues du camp, affamés. Il n'y avait plus rien du tout à manger."

Cette communautarisation particulière des Palestiniens au Liban n'a de sens et ne s'exprime que dans son rapport à la société libanaise, ce qui la singularise des autres communautés palestiniennes en exil. La constitution en communauté distincte leur a permis d'être un des acteurs du jeu politique libanais, en s'alliant ou en s'opposant aux différentes factions ou partis politiques en présence. Suite à l'invasion israélienne de 1982, les tensions avec le pouvoir libanais et la faiblesse politique et économique des réfugiés palestiniens les ont mis dans une situation marginale au sein de la société libanaise.

Les camps de réfugiés peuvent donc être vus comme des espaces de liberté et d'affirmation identitaire : ce sont les lieux de la différentiation et de l'affirmation du soi par rapport à l'autre. C'est par leur attache affirmée à une origine, leur village et à un espace, la Palestine, que les réfugiés palestiniens aspirent à jouer un rôle dans une société palestinienne à venir. C'est autour de cette articulation originale entre mémoire et rapport avec la société d'accueil que se construit l'identité palestinienne dans les camps. Le camp est un espace vivant, véritable laboratoire pour la société palestinienne à venir, qui affirme et fonde pour partie sa légitimité dans son passé, gère le présent par sa capacité d'adaptation dans les pays d'accueil et jette les bases d'un avenir commun, autour de l'idée du retour. On assiste dans ce contexte à une 
redéfinition constante des frontières de l'identité palestinienne, entre mémoire et projet politique, qui sont les deux facettes du territoire palestinien de l'exil. A la fin des années soixante c'est la lutte armée qui devient l'élément central autour duquel la mobilisation politique s'effectue.

\section{La Résistance palestinienne au service de l'affirmation de l'identité nationale.}

Les Accords du Caire ${ }^{7}$ de 1969 modifient en profondeur les relations entre l'OLP et l'Etat libanais. La centrale palestinienne obtient le droit de gérer seule l'espace des camps. Considéré comme espace mémoire et de préservation de l'identité palestinienne d'avant 1948, le camp se voit consacré comme le terreau du renouveau du nationalisme palestinien: sanctuaire naturel en terre d'accueil, dont les conditions de vie difficiles des habitants constituent, selon les termes utilisés par le FPLP lors de son troisième Congrès de mars 1972 un "terrain favorable à la révolution" [Bichara, éd., 1975 : 214], il s’impose a priori comme le point d'ancrage par excellence des organisations de la Résistance.

L'un des objectifs premiers de la Résistance palestinienne consiste à dépasser la "mentalité de réfugié" induite par l'action de l'UNRWA, dans une double perspective de mobilisation politique des réfugiés et d'affirmation de l'OLP comme incarnation et porte-parole unique du mouvement national palestinien. Par ce biais, c’est une nouvelle représentation des réfugiés, que l’OLP cherche à imposer à la communauté internationale.

\subsection{Une nouvelle représentation du réfugié}

La Résistance déclenchée par l’OLP vise à transformer la société palestinienne, et plus particulièrement les réfugiés des camps, en une société combattante et dévouée, unie par un seul et même objectif : la libération de la Palestine par la lutte armée. La transformation du Palestinien en combattant s'est effectuée par l'élaboration d'un discours stigmatisant les conditions vie misérables dans lesquelles les réfugiés des camps avaient vécu depuis leur départ de Palestine. Mais il ne s’agit plus uniquement d'éveiller la compassion de la

\footnotetext{
${ }^{7}$ Les Accord du Caire marquent la reconnaissance par les Autorités libanaises de la présence politique et armée palestinienne au Liban. Ils amènent trois principaux changements dans les relations palestino-libanaises : la communication entre les deux parties, l'encadrement de l'activité des fedayin et l'autonomisation de la gestion des camps de réfugiés.
} 
communauté internationale, comme cela avait été le cas jusque là. Révolutionnaire, le discours colporté par l’OLP vise aussi à affecter durablement la structure du pouvoir au sein des communautés des camps, en remplaçant l'élite clanique (ou tribale) traditionnelle des notables locaux par les jeunes élites nationalistes acquises aux idées de l’OLP. A cet effet, la représentation misérabiliste du réfugié des camps, à la merci des rations distribuées par l’UNRWA, s'enrichit dans les écrits politiques de la Résistance de critiques virulentes contre le système patriarcal ayant prévalu jusque là dans les camps. Jugée réactionnaire, l'élite traditionnelle est accusée d'avoir étouffé les voix rebelles de la jeunesse palestinienne et d'avoir cherché à préserver son pouvoir au prix de maintes concessions aux autorités de l'UNRWA ou à celles des pays d'accueil. C'est ainsi que les camps étaient devenus des foyers de répression interne et du terrorisme (irhâb) exercé par les services de renseignement des pays d'accueil - les moukhâbarât -, et leurs collaborateurs. C'est ce dont témoigne ce texte du Fath ${ }^{8}$ relatant le changement de mentalité des réfugiés de Jordanie et du Liban avec l'avènement de la Résistance: "Après vingt-deux ans d'oppression, d'humiliation et de manipulation par la police secrète et les exploiteurs locaux, les camps se sont éveillés à la révolution. Dans la lutte, les exilés ont brisé leurs chaînes, ils ont expulsé la police secrète, ses espions, et les exploiteurs qui étaient leurs alliés...La criminalité dans ces camps a considérablement baissé, à 10\% de ce qu'elle était avant la révolution" [Bichara, éd., 1975 : 186].

Ainsi émerge une nouvelle représentation quasi-mystique du réfugié, dont les traits se dessinent par opposition au rationnaire typique, passif et résigné. Le réfugié est désormais un combattant épris de liberté, résolu et prêt au sacrifice; un feda’iy digne, définitivement affranchi de sa dépendance économique envers l’UNRWA et de sa soumission au contrôle des Etats d'accueil. Les propos répertoriés des fedayins de tout âge témoignent d’une nécessité de reprendre leur destin en main par l'engagement dans la résistance, le retour en Palestine ne devant plus être attendu passivement à l'ombre des centres de distribution de l'UNRWA, mais être imposé a l’ennemi par l'action politique et la force des armes.

La représentation du réfugié en combattant se nourrit des faits de guerre réels ou supposés des

\footnotetext{
${ }^{8}$ L'OLP réuni plusieurs organisations politiques dont la plus influente est le Fath (acronyme inverse, en arabe, de Mouvement de Libération de la Palestine), dirigé Yasser Arafat. Ces différentes organisations, qui comptent aussi le FPLP et le FDLP, d'obédience marxiste, ou la Saïqa, d'inspiration Ba'thiste, disposent toutes d'une branche politique et d'une branche armée (tanzim).
} 
organisations de la Résistance. Les combats acharnés que livrent en mars 1968 l'armée jordanienne, les fedayins du Fath et l'Armée de Libération de la Palestine aux forces israéliennes à Karameh, dans la vallée du Jourdain, marquent les esprits : présentée de façon opportuniste comme une victoire remportée par la seule Résistance palestinienne, elle encourage des milliers de jeunes palestiniens à rejoindre les rangs des composantes de l'OLP. En Syrie de 1968 à 1970, en Jordanie en 1969-1970, puis au Liban dès 1971, des bases militaires où sont dispensés endoctrinement politique et entraînement aux armes accueillent ces jeunes dans toute zone sous son contrôle. Symboles du renouveau du nationalisme palestinien, ces camps ne constituent qu'un des éléments de l’infrastructure politico-militaire de l'OLP, qui comporte aussi des tribunaux révolutionnaires, une police militaire, des bureaux d'information et un bureau des impôts.

Fortement contesté, l’ordre social traditionnel n’est pas renversé pour autant : plusieurs études [Sayigh, 1977; Sirhan, 1971] ont souligné le rôle politique qu’il a continué à jouer dans la transmission de la mémoire de la Palestine aux nouvelles générations. Sans s'éteindre, il se voit cependant doublé puis devancé par un ordre nouveau, dont les référents se rapportent toujours à la Palestine, mais sous une forme plus opérante : les organisations de la Résistance vivifient l’opposition passive à la réinstallation, adoptée jusque là par les "milieux réfugiés", en la replaçant opiniâtrement dans le cadre d'une lutte armée de libération nationale. Le concept sacro-saint du retour prend désormais une signification supplémentaire. Il implique d’abord la ré-appropriation, en tant qu'exilé politiquement émancipé et non plus en tant que réfugié dépendant, d’une identité individuelle et collective déniée jusque là.

L'avènement de la Résistance n’inaugure pas non plus un bouleversement radical du statut des réfugiés des camps sur l'échiquier politique palestinien. Désormais représentés au niveau local par des comités populaires (lijân cha `biya) liés aux groupements de la Résistance, ils se retrouvent relégués à la base du Mouvement national. En dépit d'un discours faisant la part belle au rôle d'avant-garde de la lutte pour la libération de la Palestine et l'émancipation politique du monde arabe, qu'ils sont appelés à tenir ${ }^{9}$, les organisations de la Résistance demeurent dirigées par les membres éduqués de la petite-bourgeoisie palestinienne, politiquement marginalisée, et à la recherche d'un espace de libre expression politique

\footnotetext{
${ }^{9}$ Y compris dans la littérature palestinienne. Voir par exemple des auteurs comme Ghassan Kanafani, Mahmoud Darwish ou Ahmad Dahbour, dont l'œuvre est analysée dans les études suivantes : Al Khalili, 1997 : 26-36 ; et Jayyusi, 2000 : 86-102.
} 
soustraite aux influences des pays d'accueil et du leadership conservateur traditionnel. Comme le constate rétrospectivement un des responsables militaires du Fath en Jordanie puis au Liban: "Les réfugiés des camps ont constitué un instrument de soutien du Mouvement national, pas son instrument de commandement"10. Malgré un niveau d'instruction relativement élevé, dû en grande par les services éducatifs de l’UNRWA, ils n'en sont pas moins perçus par la nouvelle élite politique de la Résistance comme une main-d'œuvre dont il s'agit d'obtenir l'allégeance par le biais d'une éducation politique visant à les détacher de leurs éventuelles allégeances partisanes antérieures et à les amener à légitimer son rôle de guide dans la conduite de la libération de la Palestine. Le versement de soldes aux nouvelles recrues, en particulier par le Fath, a aussi été utilisé pour s’attirer les faveurs des réfugiés, devenant même, dans les années 1970 au Liban, un élément à part entière de la stratégie de survie élaborée par les réfugiés les plus défavorisés.

La mobilisation identitaire que l'OLP entreprend à travers la revalorisation de la représentation du réfugié en fedayin et la consécration de la lutte armée (al-kifâh al-musallah), n’aurait vraisemblablement pas suffi à assurer la pérennité du mouvement national palestinien. Malgré le prestige du rôle, le nombre de fedayin ne dépassa pas les 10000 et, malgré une progression des attaques à l'intérieur des frontières d'Israël, leur action armée ne permit jamais de reconquérir ne serait-ce qu’un pouce du territoire de la Palestine occupée. Tout en appelant à la lutte armée, c'est par la constitution d'un vaste réseau d'institutions sociales, que l’OLP allait parvenir à s'implanter durablement au sein des communautés de réfugiés.

\subsection{Les institutions sociales de la Résistance comme foyers d'identification nationale}

L’éclosion de la guérilla palestinienne en Jordanie ou au Liban s’est accompagnée de la mise en place d'une infrastructure civile composée de dispensaires et d'hôpitaux, de centres de formation professionnelle et d'écoles, ainsi que de bureaux d'entraide sociale. Les institutions les mieux dotées sont celles de l'OLP, dont on retrouve des ramifications dans les autres pays d'implantation, comme la Syrie, l'Egypte, l’Irak, l'Arabie Saoudite ou le Koweït. Certaines d'entre elles ont été instituées lors de la fondation de la Centrale palestinienne en 1964-1965, tels que l'Association d'aide aux familles des martyrs et détenus palestiniens, le Département

\footnotetext{
${ }^{10}$ Entretien réalisé par J. Al Husseini à Amman, février 1998.
} 
des organisations populaires réunissant les unions professionnelles, ou encore le Centre de recherches de l'OLP. D'autres furent l'initiative du Fath: c'est par exemple le cas du Croissant-Rouge palestinien, fondé en 1968 et adopté par le Conseil national palestinien l'année suivante, des écoles d'éducation politique et militaire pour garçons (lionceaux, Ashbâl) et filles (fleurs, zahrât) créées après la bataille de Karameh en 1968, ou encore de l'Association des ateliers pour les enfants de Martyrs palestiniens (SAMED). Inaugurée en 1970, cette dernière ouvrit les portes de ses 34 coopératives et ateliers en 1975-76 à l'ensemble de la population pour compter jusqu'à 3500 ouvriers en 1979 [Chesnot et Lama, 1998 : 122]. Aux institutions de l’OLP, il faut encore ajouter celles administrées de façon autonome par ses organisations membres, les fasâ'il. A la même époque celles-ci géraient au Liban une cinquantaine de cliniques, plus de quarante centres de formation et ateliers de production, une trentaine de jardins d'enfants, et plus d'une cinquantaine de clubs de jeunesse et de scouts. En tout, on estima à la veille du départ de la direction de la Résistance en 1982, que l'"économie palestinienne" contribuait pour plus de $15 \%$ au produit national brut du Liban, générant 10000 emplois directement et 30000 indirectement [Hudson, 1997 : 254]. L’œuvre sociale de la Résistance comprend aussi les importantes améliorations des conditions de vie dans les camps de réfugiés. Dès leur implantation dans ces camps, les fasâ’il ont pris en charge un certaines activités qui avaient été négligées ou supprimées par l’UNRWA faute de moyens, telles que la réhabilitation de l'habitat et la généralisation de l'électricité et de l'arrivée d'eau dans la plupart des camps en milieu urbain. Autrefois symboles de pauvreté, de passivité et de marginalisation économique, les camps deviennent ainsi le symbole de la régénérescence du peuple palestinien.

La mise en place de services sociaux autonomes constitue, après l'appel à la lutte armée, le second axe de mobilisation à travers la transformation des réfugiés en "retournants" ( ầ'idîn). Dès 1969, le Fath déclare que les services médicaux, éducatifs et sociaux de la Résistance ont “...rétabli la dignité et le respect de soi ” [Bichara, éd., 1975 : 186]. L’OLP intervient dans le même sens en 1973. C’est “...afin qu'ils obtiennent leur autonomie...” qu'elle se propose de “...s'occuper des conditions de la vie dans les camps, que ce soit sur le plan économique, social ou culturel " [Bichara, éd., 1975 : 117]. Elle tient à prouver à l'opinion publique internationale que le Palestinien "...était un être productif et non un réfugié tributaire de l'aide de l'Organisation des Nations Unies" [ONU, 1982]. Tenant compte de l'importance croissante du rôle des femmes dans la vie politique palestinienne, acquise essentiellement sous l'influence des organisations de gauche telles que le FPLP ou le FDLP, l'OLP les inclut 
officiellement comme élément à part entière dans la lutte nationale.

L’OLP avait à cœur de replacer cette renaissance institutionnelle dans une ligne historique et culturelle précise. En documentant la "question de Palestine", son Centre de recherches, créé à Beyrouth en 1965, a contribué à réunir les fragments épars d’une histoire palestinienne dont l’UNRWA d'une part, les familles de réfugiés de l'autre, avaient jusque là été les dépositaires informels. Sa revue officielle - "Chou'oun filastîniya" (Affaires palestiniennes) - et ses ressources documentaires ont aidé à reconstituer une histoire nationale dans laquelle tout Palestinien pouvait s'identifier. Cette documentation de la cause palestinienne n'est pas dénuée d'arrière-pensées politiques : elle vise à consacrer l'avènement de l'OLP comme élément majeur de cette histoire. A cet effet, elle est complétée par une série de mesures symboliques destinées à inscrire l'identité nationale dans la vie des communautés palestiniennes. Parmi ces mesures, on peut relever l'institution d'un drapeau national, inspiré de celui que brandit le Chérif Hussein de la Mecque lors de la révolte arabe contre les troupes ottomanes en 1916, l’inauguration d'un hymne national "Ma patrie, ma patrie" (bilâdi, bilâdi) et l'instauration d'un calendrier commémorant les dates marquantes de la résistance palestinienne contre le sionisme depuis le début du siècle.

\subsection{La Résistance palestinienne : un projet de société à venir ?}

Sur le plan opérationnel, les institutions de la Résistance palestinienne n’ont pas cherché à remplacer, ni même à concurrencer l’UNRWA. Celle-ci s’était déjà imposée, depuis le début de activités en mai 1950, comme un service public à part entière dans les camps. Nouvelles venues dans le domaine du social, ses institutions se sont implantées graduellement, en suppléant aux insuffisances des services de l'UNRWA et des pays d'accueil. C'est ainsi que la naissance du Croissant-Rouge palestinien correspondit aux attentes d'une population que l’infrastructure hospitalière existante, déficiente et pauvre en personnel, était incapable de satisfaire $^{11}$. Dans le domaine éducatif, les écoles du Fath ont pris en charge les laissés pour compte du système scolaire local - les enfants de martyrs en priorité - en leur dispensant une éducation basée sur celle des pays hôtes, mais où une large part est accordée à l’histoire, la

\footnotetext{
${ }^{11}$ Les camps de réfugiés, ne disposant souvent que d'un seul centre médical (dispensaire ou clinique) n’étaient visités par un médecin que deux ou trois fois dans la semaine ; ceux-ci furent vite débordés avec l'accroissement du nombre de blessés parmi les fedayins.
} 
géographie et la politique palestiniennes ${ }^{12}$. Enfin, l'OLP s'est investie opportunément dans le secteur de l'équipement (rénovation de l'habitat, de l'adduction en eau et de l'électrification des camps), délaissé par l’UNRWA et les autorités libanaises.

C'est à la faveur de la grave crise financière que connaît l'UNRWA en 1974, puis de l'écroulement de l'Etat libanais lors de la guerre civile en 1975, que les institutions civiles palestiniennes prennent une importance quasi-gouvernementale, conférant à l'OLP l'apparence d'une organisation bureaucratique hautement centralisée, d'un "Etat dans l'Etat". Mais il ne s'agit que d'une apparence : l'expansion des ces institutions ne s'est pas inscrite dans un projet collectif destiné à fonder un ordre social nouveau, pas plus qu'elle n’a été guidée par un souci de rationalisation opérationnelle. Elle a plutôt été menée par une logique de clientélisme politique auprès des réfugiés, désormais désignés comme “ ...le personnel, le staff, de la révolution" [Goichon, 1972 : 1135]. L’esprit de compétition qui a prévalu entre les différentes composantes de la Résistance a ainsi conduit à une duplication des services et une détérioration de leur qualité, quand bien même l'organisation dominante au sein de l'OLP, le Fath, s'est servie de son influence pour coopter au sein de la centrale l'ensemble des organisations concurrentes.

De plus, cette infrastructure étatique ne s'est pas fondée sur un projet de société d'avenir clairement défini. Si les dirigeants de l’OLP ont eu le mérite de mettre sur pied une infrastructure militaire et civile palestinienne malgré tout durable, montrant ainsi l'exemple pour les générations futures, ils n’ont en revanche pas réussi à formuler une vision politique et sociale cohérente à même de pouvoir fédérer l'ensemble des différentes composantes du peuple palestinien. Catapultés comme l'avant-garde de la Révolution, les réfugiés et leur direction n’ont pas réussi à transformer radicalement la structure de classe traditionnelle et à fonder un ordre social nouveau. C'est d'ailleurs une constatation que les diverses organisations de la Résistance font après la débâcle de 1970 en Jordanie, relevant, à l'instar du Fath, qu'elles avaient été incapables de se préparer à mobiliser les masses d'une manière organisée et consciente; ou encore, comme le précisa le FDLP, qu'elles s’étant contentées de fournir quelques services médicaux et sociaux et de développer quelques relations économiques, tout en se complaisant dans un "océan" de privilèges matériels et de prestiges

\footnotetext{
${ }^{12}$ Le Fath a géré trois écoles de ce type ; l’une à Amman (l'école "Jérusalem", 1968-1970) et les deux autres au Liban (les écoles modèle "Is`ad al-toufoulat" à Souk al-Gharb et "Ramallah" dans le camp de Sabra, 1970-1982).
} 
[Bichara, éd., 1975 : 169 ; 267-269].

Il convient cependant se rappeler que dans les années 1970, le mouvement national palestinien n’est pas encore mené comme une entreprise de formation étatique. Il fait encore partie de ce que E. Haas a appelé les Nation-first movements qui, à l'inverse des State-first movements, se définissent par une absence de contrôle territorial stable et de référents nationaux et territoriaux clairement établis [Amos, 1980 : 27-28]. Confiné à l'exil après ses déconvenues face à l'armée israélienne en Cisjordanie et à Gaza13, son objectif majeur est de s'assurer une base territoriale provisoire et d'imposer son pouvoir de décision et un certain degré de loyauté aux réfugiés sous son contrôle et, au-delà, dans l'ensemble de la diaspora palestinienne. La question de la formation d'un Etat palestinien n'est pas d'actualité, bien que l'on tente d'en esquisser quelques traits fondamentaux [Bichara, éd., 1975 : 183-186].

La question de l'Etat ne pouvait se poser qu'en rapport avec l'établissement d'un pouvoir national de l'OLP en Palestine. Dès 1973, l'OLP en prend le chemin lorsque ses instances législatives (Le Conseil National Palestinien) annoncent implicitement l'établissement à terme d'une Autorité nationale en Cisjordanie et à Gaza [Bichara, éd., 1975 : 126-127]; mais ne c’est qu’en décembre 1987 suite au déclenchement de l’Intifada et de la déclaration de l'Etat palestinien l'année suivante que le projet étatique palestinien prend forme et se développe institutionnellement au gré des évolutions du processus de paix actuel. A cet égard, l'expérience libanaise de l’OLP prend pour sa direction valeur de modèle.

\section{Conclusion.}

L'Organisation de Libération de la Palestine a longtemps joué un rôle central dans la structuration de la société palestinienne de la diaspora. En Jordanie dans la deuxième moitié des années soixante, puis au Liban dans les années soixante-dix, la lutte armée, associée aux figures du fedayin - le combattant palestinien -, celle du chahid - le martyr -, et de la renaissance institutionnelle palestinienne ont constitué le pivot central de la mobilisation politique et identitaire au sein du mouvement national palestinien. La prise de pouvoir des organisations de fedayin, issues des couches populaires de la société palestinienne, à la suite

\footnotetext{
13 En Cisjordanie, les tentatives d'implantation de la Résistance en 1967-1968 furent anéanties par les forces d’occupation israéliennes ; à Gaza, la résistance à l’occupation réussit à perdurer jusqu’à 1971-72.
} 
de la défaite arabe de 1967, tente de bouleverser la structure du corps social palestinien jusqu'alors dominé par les grandes familles de notables. Le conflit armé devient l'un des instruments du changement social dans les camps de l'exil, en générant une redéfinition des rapports de pouvoir au sien de la société palestinienne, avec l'émergence de nouvelles élites politiques et une production institutionnelle originale qui fait de l'OLP dans les années soixante-dix un quasi Etat sans territoire. La figure du réfugié des camps se confond avec celle du fedayin dans le discours national, et elle devient le vecteur principal de mobilisation et d'identification des Palestiniens de la diaspora. Les camps de réfugiés, et particulièrement ceux du Liban, où se concentre l'essentiel de l'infrastructure militaire palestinienne deviennent les lieux symboles de la diaspora : ils sont l'expression spatiale de l'exode de 1948, ce sont des lieux de "l'entre-soi" où se recomposent les systèmes de solidarités palestiniens.

Aujourd'hui, le retour de la direction palestinienne en Cisjordanie et à Gaza, et la volonté de création d'un Etat palestinien, entraînent une nécessaire redéfinition du rapport mouvement national / lutte armée hérité des années soixante, pour fonder une identité palestinienne qui devra se conformer à la configuration géopolitique qui émergera du processus de négociations israélo-arabes. L'intifada débutée dans les Territoire en 1987 et celle qui se développe depuis septembre 2001, ont remis sur le devant de la scène la figure symbolique du chahid, et redonné aux organisations para-militaire un rôle prépondérant sur la scène palestinienne. Quel rôle joueront alors les réfugiés des camps de l'exil dans la société palestinienne à venir ?

\section{Références :}

Al Khalili A. [1997], "Palestinian Refugees’ Image in Palestinian Literature : From Tent to Gun to Peaceful Settlement", in Majallat al-hijra al-qasriya, $1^{\text {ère }}$ année, ${ }^{\circ} 2$, hiver : 15-39.

Amos J. W. [1980], Palestinian Resistance. Organization of a Nationalist Movement. New York, Pergamon Press, $471 \mathrm{p}$.

Berque A. [1990], Médiance de milieux en paysages, Montpellier, GIP Reclus (coll. Géographiques), 163 p.

Bichara K. et Bichara N., éd. [1975], Textes de la révolution palestinienne (1968/1974), Paris, Sindbad, 350 p.

Chesnot C. et Lama J. [1998], Palestiniens 1948-1998. Génération fedayin : de la lutte armée à l’autonomie, Paris, Editions Autrement (Collection mémoires), n52, p.122. 
Ghazzawi H. [1989], "La mémoire du village et la préservation de l'identité palestinienne", in "Palestine, mémoire et territoire", Cahiers d'études stratégiques, n¹4, EHESS, CIRPES : 1340.

Goichon, A. M. [1972], Jordanie réelle (II), Paris, éd. Maisonneuve et Larose

Halbwachs M. [1950], La mémoire collective, Paris, PUF, 204 p. (édition 1968).

Hovanessian M. [1995], Les Arméniens et leurs territoires, Paris, Editions Autrement (Série monde / Français d'ailleurs, peuple d'ici), HS n 84, 173 p.

Hudson M. [1997], "Palestinians and Lebanon : the Common Story", in Journal of Refugee Studies, special issue : Palestinians in Lebanon, vol. 10, $n^{\circ} 3$, Septembre : 243-260.

Jayyusi, S. K. [2000], "La littérature palestinienne après 1948", in Revue d'études palestiniennes, ${ }^{\circ} 24$ (nouvelle série), été 2000, pp.64-104.

Médam A. [1992], "Dans l'espace-temps des Juifs", in Espaces et sociétés, nº 72 : 9-30.

Mission économique d'études des Nations Unies pour le Moyen-Orient [1949], Document A/1106, novembre, Annexe B.

ONU [1982], "Extrait du discours du porte-parole de l'OLP à la Commission politique spéciale de l'AGNU", in Documents officiels de l'Assemblée générale des Nations Unies (A/SPC/37/SR.24), Commission politique spéciale, 9 novembre 1982, paragraphe 50.

Sayigh R. [1994], Too Many Enemies. The Palestinian Experience in Lebanon, London, Zed Books Ltd, 369 p.

Sayigh R. [1977], "Sources of Palestinian Nationalism", in Journal of Palestine Studies, vol. 6 , $n^{\circ} 4$, Summer : $17-40$.

Sheffer G. [1996], "Wither the study of diaspora ? Some theorical, definitional, analytical and comparative considerations", in Georges Prévélakis (dir.) Les réseaux des diasporas. The networks of diasporas, KYKEM - L'Harmattan, Nicosie : 37-46.

Sirhan B. [1971], "Al-atfâl al-filastîniyyoun : jîl al-tahrîr", in Chou’oun filastîniyya, n¹, mars : 95-106.

UNRWA [1986], UNRWA, a brief history (1950-1982), Vienna, UNRWA Headquarters, $304 \mathrm{p}$. 